\title{
AGN-host galaxy connection: multiwavelength study
}

\author{
M. Pović ${ }^{1}$, M. Sánchez-Portal ${ }^{2}$, A. M. Pérez García ${ }^{3,4}$, \\ A. Bongiovanni ${ }^{3}$, J. Cepa ${ }^{4,3}$, and OTELO team \\ ${ }^{1}$ Instituto de Astrofísica de Andalucía (IAA-CSIC), Granada, Spain \\ email: mpovic@iaa.es \\ ${ }^{2}$ Herschel Science Centre (HSC), European Space Agency Centre (ESAC)/INSA, Villanueva \\ de la Cañada, Madrid, Spain \\ ${ }^{3}$ Instituto de Astrofísica de Canarias (IAC), La Laguna, Tenerife, Spain \\ ${ }^{4}$ Departamento de Astrofísica, Universidad de La Laguna (ULL), La Laguna, Tenerife, Spain
}

\begin{abstract}
The connection between active galactic nuclei (AGN) and their hosts showed to be important for understanding the formation and evolution of active galaxies. Using $\mathrm{X}$-ray and deep optical data, we study how morphology and colours are related to X-ray properties at redshifts $\mathrm{z} \leqslant 2.0$ for a sample of $>300 \mathrm{X}$-ray detected AGN in the Subaru/XMM-Newton Deep Survey (SXDS; Furusawa et al. 2008) and Groth-Westphal Strip (GWS; Pović et al. 2009) fields. We performed our morphological classification using the galSVM code (Huertas-Company et al. 2008), which is a new method that is particularly suited when dealing with high-redshift sources. To separate objects between X-ray unobscured and obscured, we used X-ray hardness ratio $\mathrm{HR}(0.5-2 \mathrm{keV} / 2-4.5 \mathrm{keV})$. Colour-magnitude diagrams were studied in relationship to redshift, morphology, X-ray obscuration, and X-ray-to-optical flux ratio. Around $50 \%$ of $\mathrm{X}$-ray detected AGN at $\mathrm{z} \leqslant 2.0$ analysed in this work reside in spheroidal and bulge-dominated galaxies, while at least $18 \%$ have disk-dominated hosts. This suggests that different mechanisms may be responsible for triggering the nuclear activity. When analysing populations of $\mathrm{X}$-ray detected AGN in both colour-magnitude (CMD) and colour-stellar mass diagrams (Figure 1), the highest number of sources is found to reside in the green valley at redshifts $\approx 0.5-1.5$. For the first time we studied CMD of these AGN in relation to morphology and X-ray obscuration, finding that they can reside in both early- and late-type hosts, where both morphological types cover similar ranges of $\mathrm{X}$-ray obscuration (Figure 1). Our findings appear to confirm some previous suggestions that X-ray selected AGN residing in the green valley represent a transitional population (e.g. Nandra et al. 2007, Silverman et al. 2008, Treister et al. 2009), quenching star formation by means of different AGN feedback mechanisms and evolving to red-sequence galaxies. More details on analysis and results presented here can be found in Pović et al. 2012.
\end{abstract}

Keywords. X-rays: galaxies, galaxies: active, galaxies: fundamental parameters

\section{References}

Furusawa, H. et al. 2008, ApJS, 176, 1

Huertas-Company, M. et al. 2008, A\&A A, 478, 971

Melbourne, J. et al. 2008, ApJ, 660, 81

Nandra, P. et al. 2007, ApJ, 660, L11

Pović, M. et al. 2012, A\& $A, 541,118$

Pović, M. et al. 2009, ApJ, 706, 810

Silverman, J. D. et al. 2008, ApJ, 675, 1025

Treister, E. et al. 2009, ApJ, 693, 1713

Wolf, C. et al. 2008, A\&BA, 492, 933 

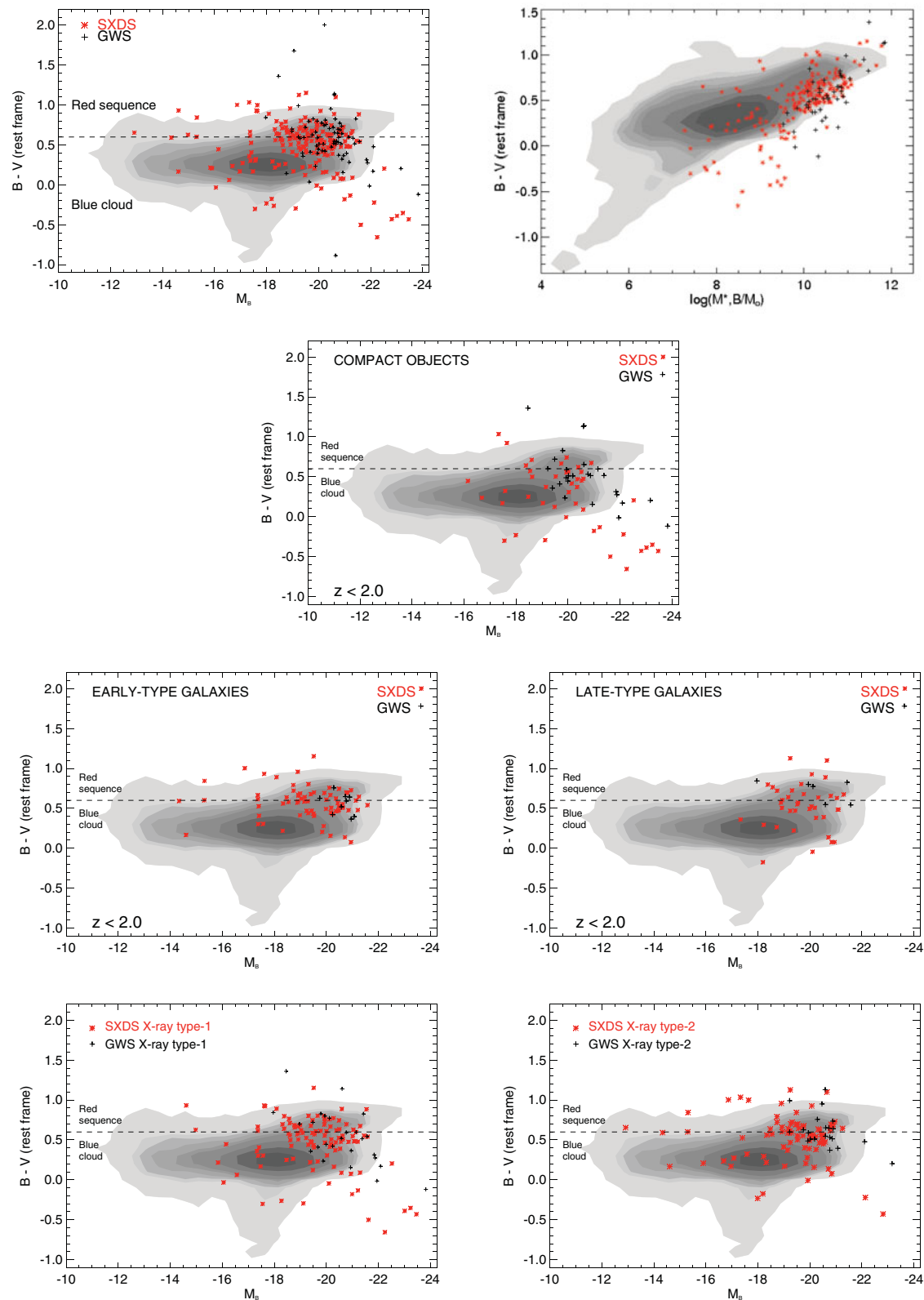

Figure 1. (Top left) Colour-magnitude diagram (CMD) showing the relation between the rest-frame $\mathrm{B}-\mathrm{V}$ colour and the absolute magnitude in the B band for a sample of AGN in the SXDS (red stars) and GWS (black crosses) fields having redshifts $\mathrm{z} \leqslant 2$. (Top right) Colour-stellar mass diagram for the same AGN and control samples as in left panel. CMDs are studied in relation with morphology for compact (middle up), early- (middle down left) and late-type (middle down right) active galaxies, and in relation with $\mathrm{X}$-ray obscuration for $\mathrm{X}$-ray unobscured (bottom left) and obscured (bottom right) sources. In all plots the sample of AGN is compared with a sample of normal galaxies from the CDF-S field (Wolf et al. 2008) represented with contours. Gray scales of the contours are scaled to the data, where the darkest and brightest show the highest and the lowest density of the sources, respectively. The dashed line shows the Melbourne et al. (2007) separation between the red sequence $(\mathrm{B}-\mathrm{V}>0.6)$ and blue cloud $(\mathrm{B}-\mathrm{V}<0.6)$ galaxies. 